\title{
Hyperreal-Valued Probability Measures Approximating a Real-Valued Measure
}

\author{
Thomas Hofweber and Ralf Schindler
}

\begin{abstract}
We give a direct and elementary proof of the fact that every realvalued probability measure can be approximated—up to an infinitesimal—by a hyperreal-valued one which is regular and defined on the whole powerset of the sample space.
\end{abstract}

When we measure the probability of events, we assign numbers to these events in accordance to how likely they are. Standard probability theory assigns real numbers to events, but there are well-known problems with using real numbers as the measures of probability. One of them is that measure 0 events do not form a homogeneous class; that is to say, there seem to be differences in probability among events which get assigned the same measure of their probability, namely, the lowest possible measure 0 . To illustrate with a standard example, let $\Omega$ be any nonempty set. Let us randomly pick an element of $\Omega$. What is the chance that a given element $a \in \Omega$ gets chosen? If $\Omega$ is finite, then the answer should be $\frac{1}{n}$, where $n$ is the number of elements of $\Omega$. But what if $\Omega$ is infinite? If the measure of probability is a real number between 0 and 1 , then the answer has to be 0 , since it should be lower than $\frac{1}{n}$ for each $n$. But 0 is also the measure of the probability of the impossible event of $a$ being picked as well as not picked. These events seem to differ in their probability, since one of them might well be the one that happens, while the other one for sure will not.

To measure probability in a way that respects this difference we thus need to employ numbers other than the real numbers as measures of probability. The reason for the failure of real numbers to be able to measure probability fine enough to respect these differences is, in the end, that real numbers have the Archimedean property: Any positive real number, no matter how small, is still larger than some $\frac{1}{n}, n \in \mathbb{N}$.

Received March 22, 2013; accepted January 6, 2014

First published online April 6, 2016

2010 Mathematics Subject Classification: Primary 60A; Secondary $20 \mathrm{E}$

Keywords: probability, hyperreal numbers, measure theory

(C) 2016 by University of Notre Dame 10.1215/00294527-3542210 


\title{
Hyperreal-Valued Probability Measures Approximating a Real-Valued Measure
}

\author{
Thomas Hofweber and Ralf Schindler
}

\begin{abstract}
We give a direct and elementary proof of the fact that every realvalued probability measure can be approximated—up to an infinitesimal—by a hyperreal-valued one which is regular and defined on the whole powerset of the sample space.
\end{abstract}

When we measure the probability of events, we assign numbers to these events in accordance to how likely they are. Standard probability theory assigns real numbers to events, but there are well-known problems with using real numbers as the measures of probability. One of them is that measure 0 events do not form a homogeneous class; that is to say, there seem to be differences in probability among events which get assigned the same measure of their probability, namely, the lowest possible measure 0 . To illustrate with a standard example, let $\Omega$ be any nonempty set. Let us randomly pick an element of $\Omega$. What is the chance that a given element $a \in \Omega$ gets chosen? If $\Omega$ is finite, then the answer should be $\frac{1}{n}$, where $n$ is the number of elements of $\Omega$. But what if $\Omega$ is infinite? If the measure of probability is a real number between 0 and 1 , then the answer has to be 0 , since it should be lower than $\frac{1}{n}$ for each $n$. But 0 is also the measure of the probability of the impossible event of $a$ being picked as well as not picked. These events seem to differ in their probability, since one of them might well be the one that happens, while the other one for sure will not.

To measure probability in a way that respects this difference we thus need to employ numbers other than the real numbers as measures of probability. The reason for the failure of real numbers to be able to measure probability fine enough to respect these differences is, in the end, that real numbers have the Archimedean property: Any positive real number, no matter how small, is still larger than some $\frac{1}{n}, n \in \mathbb{N}$.

Received March 22, 2013; accepted January 6, 2014

First published online April 6, 2016

2010 Mathematics Subject Classification: Primary 60A; Secondary $20 \mathrm{E}$

Keywords: probability, hyperreal numbers, measure theory

(C) 2016 by University of Notre Dame 10.1215/00294527-3542210 


\title{
Hyperreal-Valued Probability Measures Approximating a Real-Valued Measure
}

\author{
Thomas Hofweber and Ralf Schindler
}

\begin{abstract}
We give a direct and elementary proof of the fact that every realvalued probability measure can be approximated—up to an infinitesimal—by a hyperreal-valued one which is regular and defined on the whole powerset of the sample space.
\end{abstract}

When we measure the probability of events, we assign numbers to these events in accordance to how likely they are. Standard probability theory assigns real numbers to events, but there are well-known problems with using real numbers as the measures of probability. One of them is that measure 0 events do not form a homogeneous class; that is to say, there seem to be differences in probability among events which get assigned the same measure of their probability, namely, the lowest possible measure 0 . To illustrate with a standard example, let $\Omega$ be any nonempty set. Let us randomly pick an element of $\Omega$. What is the chance that a given element $a \in \Omega$ gets chosen? If $\Omega$ is finite, then the answer should be $\frac{1}{n}$, where $n$ is the number of elements of $\Omega$. But what if $\Omega$ is infinite? If the measure of probability is a real number between 0 and 1 , then the answer has to be 0 , since it should be lower than $\frac{1}{n}$ for each $n$. But 0 is also the measure of the probability of the impossible event of $a$ being picked as well as not picked. These events seem to differ in their probability, since one of them might well be the one that happens, while the other one for sure will not.

To measure probability in a way that respects this difference we thus need to employ numbers other than the real numbers as measures of probability. The reason for the failure of real numbers to be able to measure probability fine enough to respect these differences is, in the end, that real numbers have the Archimedean property: Any positive real number, no matter how small, is still larger than some $\frac{1}{n}, n \in \mathbb{N}$.

Received March 22, 2013; accepted January 6, 2014

First published online April 6, 2016

2010 Mathematics Subject Classification: Primary 60A; Secondary $20 \mathrm{E}$

Keywords: probability, hyperreal numbers, measure theory

(C) 2016 by University of Notre Dame 10.1215/00294527-3542210 


\title{
Hyperreal-Valued Probability Measures Approximating a Real-Valued Measure
}

\author{
Thomas Hofweber and Ralf Schindler
}

\begin{abstract}
We give a direct and elementary proof of the fact that every realvalued probability measure can be approximated—up to an infinitesimal—by a hyperreal-valued one which is regular and defined on the whole powerset of the sample space.
\end{abstract}

When we measure the probability of events, we assign numbers to these events in accordance to how likely they are. Standard probability theory assigns real numbers to events, but there are well-known problems with using real numbers as the measures of probability. One of them is that measure 0 events do not form a homogeneous class; that is to say, there seem to be differences in probability among events which get assigned the same measure of their probability, namely, the lowest possible measure 0 . To illustrate with a standard example, let $\Omega$ be any nonempty set. Let us randomly pick an element of $\Omega$. What is the chance that a given element $a \in \Omega$ gets chosen? If $\Omega$ is finite, then the answer should be $\frac{1}{n}$, where $n$ is the number of elements of $\Omega$. But what if $\Omega$ is infinite? If the measure of probability is a real number between 0 and 1 , then the answer has to be 0 , since it should be lower than $\frac{1}{n}$ for each $n$. But 0 is also the measure of the probability of the impossible event of $a$ being picked as well as not picked. These events seem to differ in their probability, since one of them might well be the one that happens, while the other one for sure will not.

To measure probability in a way that respects this difference we thus need to employ numbers other than the real numbers as measures of probability. The reason for the failure of real numbers to be able to measure probability fine enough to respect these differences is, in the end, that real numbers have the Archimedean property: Any positive real number, no matter how small, is still larger than some $\frac{1}{n}, n \in \mathbb{N}$.

Received March 22, 2013; accepted January 6, 2014

First published online April 6, 2016

2010 Mathematics Subject Classification: Primary 60A; Secondary $20 \mathrm{E}$

Keywords: probability, hyperreal numbers, measure theory

(C) 2016 by University of Notre Dame 10.1215/00294527-3542210 


\title{
Hyperreal-Valued Probability Measures Approximating a Real-Valued Measure
}

\author{
Thomas Hofweber and Ralf Schindler
}

\begin{abstract}
We give a direct and elementary proof of the fact that every realvalued probability measure can be approximated—up to an infinitesimal—by a hyperreal-valued one which is regular and defined on the whole powerset of the sample space.
\end{abstract}

When we measure the probability of events, we assign numbers to these events in accordance to how likely they are. Standard probability theory assigns real numbers to events, but there are well-known problems with using real numbers as the measures of probability. One of them is that measure 0 events do not form a homogeneous class; that is to say, there seem to be differences in probability among events which get assigned the same measure of their probability, namely, the lowest possible measure 0 . To illustrate with a standard example, let $\Omega$ be any nonempty set. Let us randomly pick an element of $\Omega$. What is the chance that a given element $a \in \Omega$ gets chosen? If $\Omega$ is finite, then the answer should be $\frac{1}{n}$, where $n$ is the number of elements of $\Omega$. But what if $\Omega$ is infinite? If the measure of probability is a real number between 0 and 1 , then the answer has to be 0 , since it should be lower than $\frac{1}{n}$ for each $n$. But 0 is also the measure of the probability of the impossible event of $a$ being picked as well as not picked. These events seem to differ in their probability, since one of them might well be the one that happens, while the other one for sure will not.

To measure probability in a way that respects this difference we thus need to employ numbers other than the real numbers as measures of probability. The reason for the failure of real numbers to be able to measure probability fine enough to respect these differences is, in the end, that real numbers have the Archimedean property: Any positive real number, no matter how small, is still larger than some $\frac{1}{n}, n \in \mathbb{N}$.

Received March 22, 2013; accepted January 6, 2014

First published online April 6, 2016

2010 Mathematics Subject Classification: Primary 60A; Secondary $20 \mathrm{E}$

Keywords: probability, hyperreal numbers, measure theory

(C) 2016 by University of Notre Dame 10.1215/00294527-3542210 


\title{
Hyperreal-Valued Probability Measures Approximating a Real-Valued Measure
}

\author{
Thomas Hofweber and Ralf Schindler
}

\begin{abstract}
We give a direct and elementary proof of the fact that every realvalued probability measure can be approximated—up to an infinitesimal—by a hyperreal-valued one which is regular and defined on the whole powerset of the sample space.
\end{abstract}

When we measure the probability of events, we assign numbers to these events in accordance to how likely they are. Standard probability theory assigns real numbers to events, but there are well-known problems with using real numbers as the measures of probability. One of them is that measure 0 events do not form a homogeneous class; that is to say, there seem to be differences in probability among events which get assigned the same measure of their probability, namely, the lowest possible measure 0 . To illustrate with a standard example, let $\Omega$ be any nonempty set. Let us randomly pick an element of $\Omega$. What is the chance that a given element $a \in \Omega$ gets chosen? If $\Omega$ is finite, then the answer should be $\frac{1}{n}$, where $n$ is the number of elements of $\Omega$. But what if $\Omega$ is infinite? If the measure of probability is a real number between 0 and 1 , then the answer has to be 0 , since it should be lower than $\frac{1}{n}$ for each $n$. But 0 is also the measure of the probability of the impossible event of $a$ being picked as well as not picked. These events seem to differ in their probability, since one of them might well be the one that happens, while the other one for sure will not.

To measure probability in a way that respects this difference we thus need to employ numbers other than the real numbers as measures of probability. The reason for the failure of real numbers to be able to measure probability fine enough to respect these differences is, in the end, that real numbers have the Archimedean property: Any positive real number, no matter how small, is still larger than some $\frac{1}{n}, n \in \mathbb{N}$.

Received March 22, 2013; accepted January 6, 2014

First published online April 6, 2016

2010 Mathematics Subject Classification: Primary 60A; Secondary $20 \mathrm{E}$

Keywords: probability, hyperreal numbers, measure theory

(C) 2016 by University of Notre Dame 10.1215/00294527-3542210 